ten jetzt fest in den klinischen Alltag integriert wird. Für unser Symposium, zu dem wir Sie herzlich einladen, haben wir zu den wichtigsten Themen Vorträge vorbereitet. Wir wollen jedoch mit Ihnen intensiv diskutieren, wie die Integration des neuen Verfahrens in das onkologische Gesamtkonzept gelingen kann.

Forum 2016 $31: 520$

DOI 10.1007/s12312-016-0176-8

Online publiziert: 10. November 2016

(c) Springer-Verlag Berlin Heidelberg 2016
Auf eine spannende Veranstaltung freuen sich Prof. U. Keilholz, Dr. A. Schmittel und Prof. P. Feyer.

Wann: 14. Dezember 2016, ab 17.00 Uhr

Wo: Berliner Krebsgesellschaft e. V., Kaiserin Friedrich-Haus Robert-Koch-Platz 7, 10115 Berlin

\section{Kontakt}

Berliner Krebsgesellschaft e.V.

Tel.: 0302832400

E-Mail: info@berliner-krebsgesellschaft.de www.berliner-krebsgesellschaft.de

\title{
Jung und Diagnose Krebs
}

\author{
Neue Selbsthilfegruppe gegründet
}

Jung und Diagnose Krebs - eine Situation, in der die Pläne und Hoffnungen vieler Betroffenen plötzlich zerplatzen und die Welt ins Wanken gerät. Wo kann man auf Unterstützung, auf Verständnis und Rat hoffen?

Die Saarländische Krebsgesellschaft e. V. freut sich über die neu ins Leben gerufene Selbsthilfegruppe „JUNG \& DIAGNOSE KREBS“, in der betroffene junge Patienten aus dem Saarland in der Gruppe zusammenkommen, sich über ihr „neues Leben“ austauschen und über
Therapien, über gute und schlechte Tage, über die Zukunft und alles, was ihnen sonst noch auf dem Herzen liegt, reden können.

Die Gruppe richtet sich an Betroffene bis 35 Jahre und trifft sich ab dem 26. Oktober 2016 jeden 4. Mittwoch im Monat, von 19-21 Uhr. Treffpunkt sind die Geschäftsräume der Saarländischen Krebsgesellschaft e. V., Sulzbachstr. 37, 66111 Saarbrücken. Anfragen über info@saarlaendische-krebsgesellschaft.de oder telefonisch unter 0681-95906673.

\section{Kontakt}

Saarländische Krebsgesellschaft e.V. Beratungsstelle für an Krebs erkrankte Menschen und Angehörige Sulzbachstraße 37

66111 Saarbrücken 068195906673 (Zentrale) info@saarlaendische-krebsgesellschaft.de www.saarlaendische-krebsgesellschaft.de 